\title{
Direct Fabrication of Carbon Nanotubes STM Tips by Liquid Catalyst-Assisted Microwave Plasma-Enhanced Chemical Vapor Deposition
}

\author{
Fa-Kuei Tung, Masamichi Yoshimura, and Kazuyuki Ueda \\ Nano High-Tech Research Center, Toyota Technological Institute, 2-12-1 Hisakata, Tempaku-ku, Nagoya 468-8511, Japan \\ Correspondence should be addressed to Masamichi Yoshimura, yoshi@toyota-ti.ac.jp
}

Received 4 November 2008; Accepted 30 December 2008

Recommended by Rakesh Joshi

Direct and facile method to make carbon nanotube (CNT) tips for scanning tunneling microscopy (STM) is presented. Cobalt (Co) particles, as catalysts, are electrochemically deposited on the apex of tungsten (W) STM tip for CNT growth. It is found that the quantity of Co particles is well controlled by applied DC voltage, concentration of catalyst solution, and deposition time. Using optimum growth condition, CNTs are successfully synthesized on the tip apex by catalyst-assisted microwave-enhanced chemical vapor deposition (CA-MPECVD). A HOPG surface is clearly observed at an atomic scale using the present CNT-STM tip.

Copyright ( 92009 Fa-Kuei Tung et al. This is an open access article distributed under the Creative Commons Attribution License, which permits unrestricted use, distribution, and reproduction in any medium, provided the original work is properly cited.

\section{Introduction}

Carbon nanotube (CNT) [1] has attracted considerable attention because of their unique and outstanding properties, such as chemical inertness, small tip radius, highaspect ratio, mechanical robustness, and high electrical conductivity. It is demonstrated that the CNTs are suitable as tips of atomic force microscopy (AFM) [2-4] and scanning tunneling microscopy (STM) $[5,6]$. Numerous methods to make CNTs tips have been reported: manual attachment of CNTs to the tip apex using an acrylic adhesive [2], electron beam-induced deposition (EBID) of amorphous carbon under SEM [7], and direct growth by a chemical vapor deposition (CVD) technique [8-12]. The CVD technique is most preferred in terms of mass production in the commercial use. Although a single CNT growth is ideally suitable for CNT tip in scanning probe microscopy (SPM), plenty of CNTs are grown over the whole tip by CVD because of the difficulty in positioning catalyst only at the tip apex [12].

In the present study, we have developed fast- and high-reproducible fabrication method of CNT-STM tips. Liquid catalyst-assisted microwave plasma-enhanced CVD (MPECVD) has been used to fabricate highly aligned CNTs onto the STM tip apex. Catalytic particles are deposited only at the STM tip apex by this electrochemical method. It is shown that the quantity of deposited catalyst particles is well controlled by tip immersion time, applied DC voltage, and solution concentration. Using an optimized growth condition, a single CNT is successfully grown onto the STM tip. The performance of the present CNT-STM tip is demonstrated by the observation of HOPG surface.

\section{Experimental}

A tungsten (W) tip $(0.25 \mathrm{~mm}$ in diameter) for STM was prepared by electrochemical etching in $\mathrm{KOH}$ solution $(2.5 \mathrm{~N})$. The catalytic solution was prepared from pure water, liquid ammonia, 2-ethylhexanoicacid, and cobalt chloride with different concentrations as $10^{-1} \mathrm{M}, 10^{-2} \mathrm{M}$, and $10^{-3} \mathrm{M}$. After a standstill for a couple of hours, the mixture separated into two gravity-stabilized layers, and the topmost transparent layer was used as catalytic solution. The prepared $\mathrm{W}$ tip was immersed in the solution by a micrometer-adjustable apparatus. The different DC voltages $(2 \mathrm{~V}, 4 \mathrm{~V}$, and $8 \mathrm{~V})$ were applied on $\mathrm{W}$ tips to control the number of catalytic particles attached on the tip apex and to investigate aggregation of catalytic particles on $\mathrm{W}$ tips. The, thus, prepared catalyst covered $\mathrm{W}$ tip was placed into a 


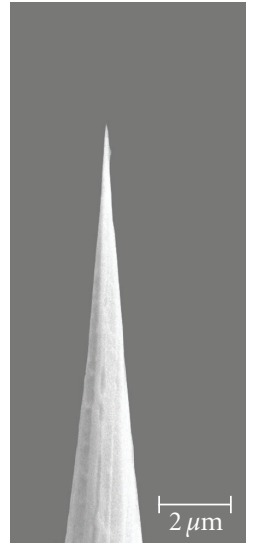

(a)

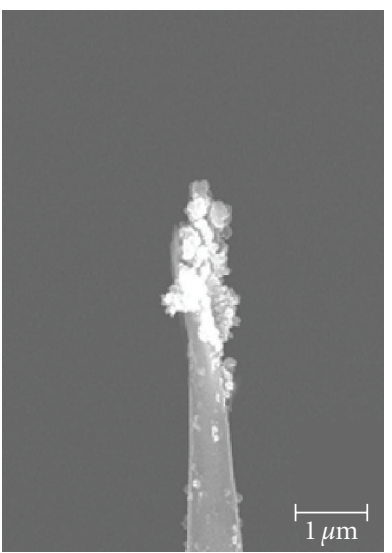

(d)

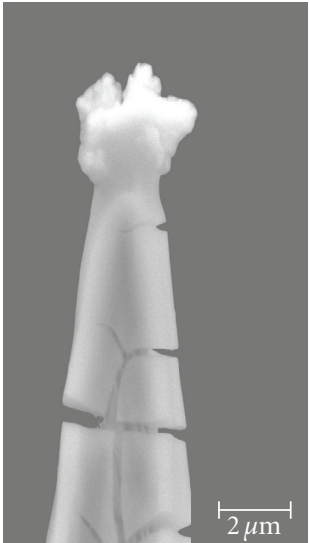

(b)

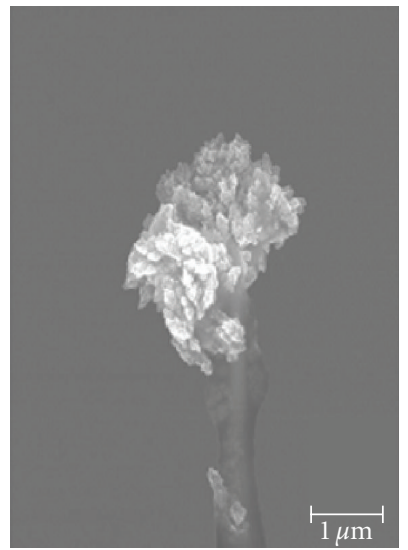

(e)

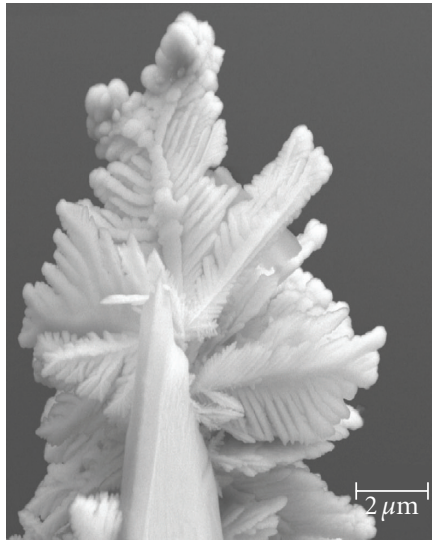

(c)

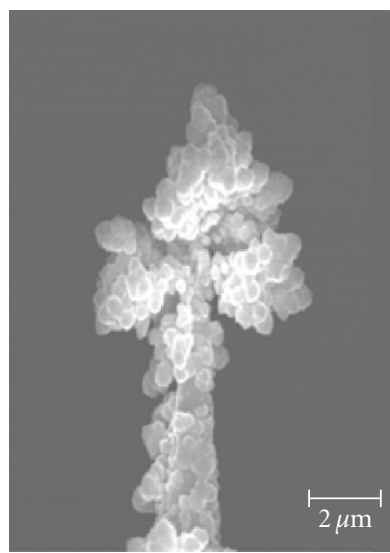

(f)

FIGURE 1: SEM pictures of catalyst-deposited tungsten tips with different electrochemical conditions. (a) voltage (4 V), concentration $(0.05 \mathrm{M}$ ), and time (1 second); (b) $4 \mathrm{~V}, 0.05 \mathrm{M}$, and 5 seconds; (c) $8 \mathrm{~V}, 0.05 \mathrm{M}$, and 5 seconds; (d) $2 \mathrm{~V}, 0.001 \mathrm{M}$, and 5 seconds; (e) $2 \mathrm{~V}$, $0.01 \mathrm{M}$, and 5 seconds; (d) $2 \mathrm{~V}, 0.1 \mathrm{M}$, and 5 seconds.

custom-made tip holder in a commercial MPECVD system (CVD-CN-100, Ulvac Japan, Kanagawa, Japan). The tip holder is designed so that the damage by plasma can be minimized during CNTs growth. Prior to CNTs growth, the tips were exposed to hydrogen plasma for the formation of catalyst particles as well as catalyst activation. Subsequently, methane and hydrogen gases were introduced for the CNTs growth. The mass flow ratio of $\mathrm{CH}_{4}: \mathrm{H}_{2}$ was kept at 1:4. The growth pressure was kept in the range of 1-5 torr. The microwave generator provides $2.45 \mathrm{GHz}, 500 \mathrm{~W}$ microwave. The growth time was 5-10 minutes, and a DC voltage of $-200 \mathrm{~V}$ was applied to the tip. The temperature of sample holder during CNTs growth process measured by a K-type thermocouple was about $700^{\circ} \mathrm{C}$. Field emission scanning electron microscopy (FESEM, Hitachi S-4700) was used to characterize the attached quantity of catalyst particles and the CNTs structures. The crystallization of CNTs was studied by using a Raman spectroscopy (Jobin Yvon HR-800) with a $633(1.96 \mathrm{eV})$ wavelength laser and the analysis power of $25 \mathrm{~mW}$. STM measurement of a highly oriented pyrolytic graphite (HOPG) surface was performed in air to examine the capability of the CNTs tip prepared in this study.

\section{Results and Discussion}

The aggregation of catalyst particles on $\mathrm{W}$ tip apex was investigated with different deposition parameters. The effect of deposition time on catalytic aggregation was shown in Figure 1(a), and Figure 1(b) shows SEM images after deposition for 1 second (a) and 5 seconds (b), with immersing depth of $0.01 \mathrm{~mm}$, solution concentration of $0.05 \mathrm{M}$, and applied voltage of 4 V. In Figure 1(a), no catalyst particles can be observed, while apparent catalyst particles are observed in Figure 1(b). This result suggests that the quantity of catalyst particles on the $\mathrm{W}$ tip increases with deposition time. Figures 1(b) and 1(c) show the effect of applied voltage on the aggregation of catalytic particles. Other deposition parameters are kept constant: immersing depth $(0.01 \mathrm{~mm})$, catalytic concentration $(0.05 \mathrm{M})$, and deposited time (5 seconds). The effect of solution concentration on catalytic aggregation was shown in Figures $1(\mathrm{~d})-1(\mathrm{f})$, where other deposition parameters are kept constant: immersing depth $(0.1 \mathrm{~mm})$, applied voltage $(2 \mathrm{~V})$, and deposited time (5 seconds). The higher solution concentration gives the larger size of deposited materials. 




(a)

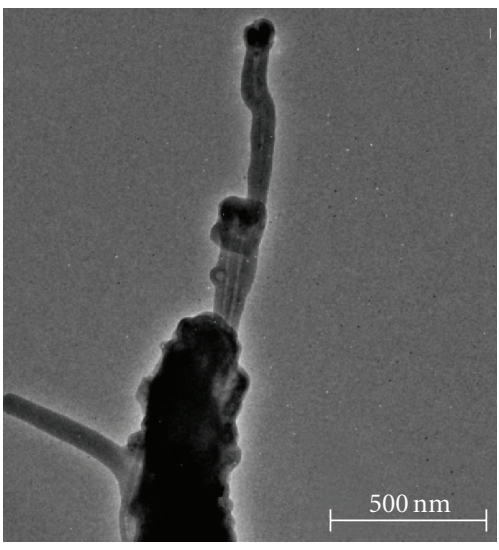

(c)

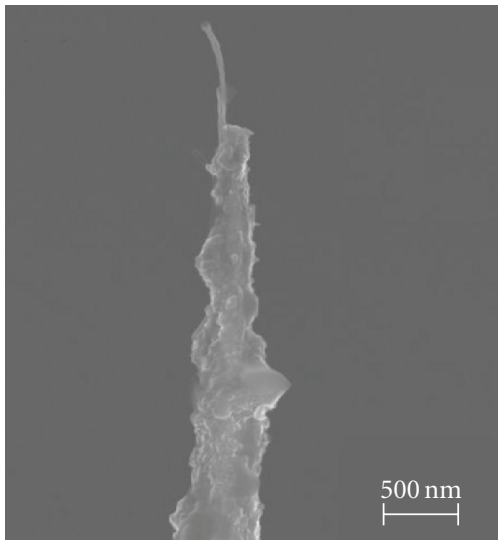

(b)

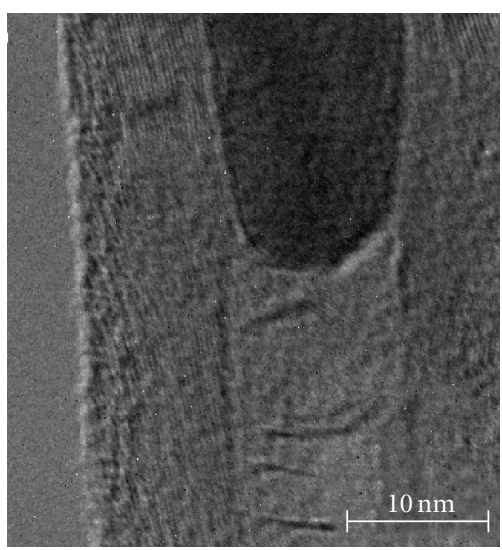

(d)

Figure 2: CNT-STM tips fabricated at the apex of tungsten tip. (a) CNTs from the deposited tip of Figure 1(d). (b) SEM and ((c), (d)) TEM images grown at the apex with optimum condition $(2 \mathrm{~V}, 0.001 \mathrm{M}$, and $\sim 0.5$ seconds).

Co cationic particles in solvent are accelerated by electronic force forward to cathode $\mathrm{W}$ tip apex. The amount of deposited aggregation particles has been shown to be positively correlated with Co cationic particles flux, then, the relationship between relative parameters and cationic particles can be supposed as follows:

$$
\begin{gathered}
N \propto A * S * F, \\
F \propto M * V,
\end{gathered}
$$

where $N$ denotes amount of deposited aggregation particles, $F$ denotes flux of ionic particles in unit time, $A$ denotes deposition area perpendicular to flux, $S$ denotes deposition time, $M$ denotes concentration of ion solvent, and $V$ denotes applied bias voltage.

Our experimental results can easily prove this postulate. Higher applied voltage enhances the more ionic particles passing through the unit area in a constant time, that is, the ion flux is increasing, further increases the deposited aggregation particles. In the same concentration and applied voltage, the ion flux in one unit area is in constant, then, the longer deposition time allows more ion particles to pass through the unit area, resulting in the increased deposition of particles. Finally, the higher concentration also increases the ion flux in unit area in a constant time and enhances the particles deposition. Those results allow us to assume that catalyst particles aggregation on $\mathrm{W}$ tip increases with the increasing of the ionic flux. Thus, the amount of deposited ionic particles can be controlled by the ion flux and depositing time. Besides, in this comparison, the overdeposition cases were employed in order to emphasize the relationship between different applied parameters and the catalyst particles aggregations on the $\mathrm{W}$ tip. Figure 2(a) shows CNTs grown on the catalyst-coated tungsten tip as shown in Figure $1(\mathrm{~d})(2 \mathrm{~V}, 0.001 \mathrm{M}$, and 5 seconds). Numerous CNTs are grown on the tip surface, indicating the presence of catalytic Co particles using the present deposition technique.

Figure 2(b) shows low-magnification SEM image of the $\mathrm{W}$ tip apex which was deposited by applied bias $2 \mathrm{~V}$, catalytic concentration $(0.001 \mathrm{M})$, and deposited time ( $\sim 0.5$ second) after CNT growth. Figures 2(c) and 2(d) show TEM images of the same tip. The diameter and length are $\sim 40 \mathrm{~nm}$ and $\sim 900 \mathrm{~nm}$, respectively. The number of graphen layers is 20 30. The CNT growth mechanism is clearly confirmed as tip growth [13], since the Co particle can be observed at the end 


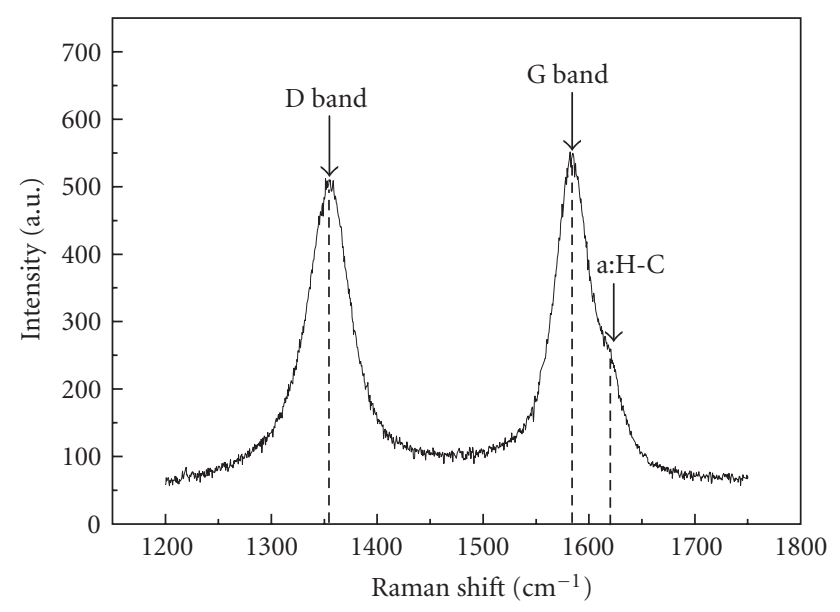

FIgURE 3: The baseline-treated Raman spectrum of CNT tip. In addition to graphite structure ( $\mathrm{G}$ band) and disordered carbon structure ( $\mathrm{D}$ band), amorphous hydrogenated carbon structure (a:H-C) is also observed.


FIGURE 4: STM image of HOPG surface obtained at a bias voltage of $6.2 \mathrm{mV}$. Honeycomb structure is clearly resolved.

of the CNTs. As previously shown, the CNTs of $50 \mathrm{~nm}$ to $80 \mathrm{~nm}$ in diameter and $\sim 800 \mathrm{~nm}$ to $1000 \mathrm{~nm}$ in length can be controlled by growth time [12].

Raman spectra are taken to spectroscopically characterize CNTs grown. The baseline-corrected Raman spectrum from CNT tip is shown in Figure 3. After using three Lorentzian peaks fitting, the center positions were specified at $1354 \mathrm{~cm}^{-1}, 1584 \mathrm{~cm}^{-1}$, and $1619 \mathrm{~cm}^{-1}$. The intensity rate and area rate were 5.47:5.84:1 and 12.51:11.03:1, respectively. The peak at $1354 \mathrm{~cm}^{-1}$ as defined as D band [14] and high $\mathrm{D}$ band shows the amorphous carbon structure similar with active coal. The peak at $1584 \mathrm{~cm}^{-1}$ is defined as $\mathrm{G}$ band, and it shows the graphite structure of CNTs similar with polycrystalline graphite powder [14]. The high intensity $I_{\mathrm{D}} / I_{\mathrm{G}}$ ratio shows the structure defects of CNTs which were synthesized by catalyst pyrolytic method [15]. Peak at $1619 \mathrm{~cm}^{-1}$ comes from amorphous hydrogenated carbon (a:H-C) [16], which is regarded as one kind of defective graphite structure.

In order to demonstrate the capability of the CNT tip fabricated, highly-ordered pyrolytic graphite (HOPG) surface was observed as a test sample. Figure 4 shows the STM image taken in air. A honeycomb structure of graphite was obviously resolved at a bias voltage of $6.2 \mathrm{mV}$, although the image is somewhat distorted due to thermal drift. The corrugation measured is about $0.2 \mathrm{~nm}$, comparable to the value obtained by conventional STM tips. However, we believe the present CNT tip becomes more powerful in the characterization of deep trench-like structures because of its high aspect ratio.

\section{Conclusion}

We have succeeded in fabricating the CNT-STM tip by liquid catalyst-assisted MPECVD. Highly aligned CNT with 30$50 \mathrm{~nm}$ in diameter was grown on STM tip apex, as revealed by FE-SEM and TEM. We have demonstrated the performance of those STM tips by obtaining atomic images of the HOPG surface. This method allows us to place and deposit catalytic particles in a controlled way on the tip apex much easier than conventional methods. It should be noted that even a single CNT-STM tip can be fabricated by our CA-MPECVD method.

\section{Acknowledgments}

This work is supported by "High-Tech Research Center" project for private universities: matching fund subsidy from Ministry of Education, Culture, Sports, Science and Technology (MEXT), 2006-2008. The authors also would like to thank Professor Hisanori Shinohara and Mr. Yuya Kamizono of Nagoya University for Raman spectra measurements, Professor Takayoshi Tanji, and Mr. Yutaka Ohira for TEM observation.

\section{References}

[1] S. Iijima, "Helical microtubules of graphitic carbon," Nature, vol. 354 , no. 6348 , pp. 56-58, 1991.

[2] H. Dai, J. H. Hafner, A. G. Rinzler, D. T. Colbert, and R. E. Smalley, "Nanotubes as nanoprobes in scanning probe microscopy," Nature, vol. 384, no. 6605, pp. 147-150, 1996.

[3] S. S. Wong, A. T. Woolley, T. W. Odom, et al., "Single-walled carbon nanotube probes for high-resolution nanostructure imaging," Applied Physics Letters, vol. 73, no. 23, pp. 34653467, 1998.

[4] J. H. Hafner, C.-L. Cheung, T. H. Oosterkamp, and C. M. Lieber, "High-yield assembly of individual single-walled carbon nanotube tips for scanning probe microscopies," Journal of Physical Chemistry B, vol. 105, no. 4, pp. 743-746, 2001.

[5] M. Ishikawa, M. Yoshimura, and K. Ueda, "A study of friction by carbon nanotube tip," Applied Surface Science, vol. 188, no. 3-4, pp. 456-459, 2002.

[6] M. Ishikawa, M. Yoshimura, and K. Ueda, "Carbon nanotube as a probe for friction force microscopy," Physica B, vol. 323, no. 1-4, pp. 184-186, 2002.

[7] H. Nishijima, S. Kamo, S. Akita, et al., "Carbon-nanotube tips for scanning probe microscopy: preparation by a controlled process and observation of deoxyribonucleic acid," Applied Physics Letters, vol. 74, no. 26, pp. 4061-4063, 1999. 
[8] C. L. Cheung, J. H. Hafner, T. W. Odom, K. Kim, and C. M. Lieber, "Growth and fabrication with single-walled carbon nanotube probe microscopy tips," Applied Physics Letters, vol. 76, no. 21, pp. 3136-3138, 2000.

[9] E. Yenilmez, Q. Wang, R. J. Chen, D. Wang, and H. Dai, "Wafer scale production of carbon nanotube scanning probe tips for atomic force microscopy," Applied Physics Letters, vol. 80, no. 12, p. 2225, 2002.

[10] Y. Shingaya, T. Nakayama, and M. Aono, "Carbon nanotube tip for scanning tunneling microscopy," Physica B, vol. 323, no. 1-4, pp. 153-155, 2002.

[11] F. M. Pan, Y. B. Liu, Y. Chang, et al., "Selective growth of carbon nanotube on scanning probe tips by microwave plasma chemical vapor deposition," Journal of Vacuum Science and Technology B, vol. 22, no. 1, pp. 90-93, 2004.

[12] M. Yoshimua, S. Jo, and K. Ueda, "Fabrication of carbon nanostructure onto the apex of scanning tunneling microscopy probe by chemical vapor deposition," Japanese Journal of Applied Physics, vol. 42, no. 7B, pp. 4841-4843, 2003.

[13] S. B. Sinnott, R. Andrews, D. Qian, et al., "Model of carbon nanotube growth through chemical vapor deposition," Chemical Physics Letters, vol. 315, no. 1-2, pp. 25-30, 1999.

[14] M. J. Matthews, M. A. Pimenta, G. Dresselhaus, M. S. Dresselhaus, and M. Endo, "Origin of dispersive effects of the Raman D band in carbon materials," Physical Review B, vol. 59, no. 10, pp. R6585-R6588, 1999.

[15] P. Tan, S.-L. Zhang, K. T. Yue, et al., "Comparative Raman study of carbon nanotubes prepared by D.C. arc discharge and catalytic methods," Journal of Raman Spectroscopy, vol. 28, no. 5, pp. 369-372, 1997.

[16] N. Boutroy, Y. Pernel, J. M. Rius, et al., "Hydrogenated amorphous carbon film coating of PET bottles for gas diffusion barriers," Diamond and Related Materials, vol. 15, no. 4-8, pp. 921-927, 2006. 

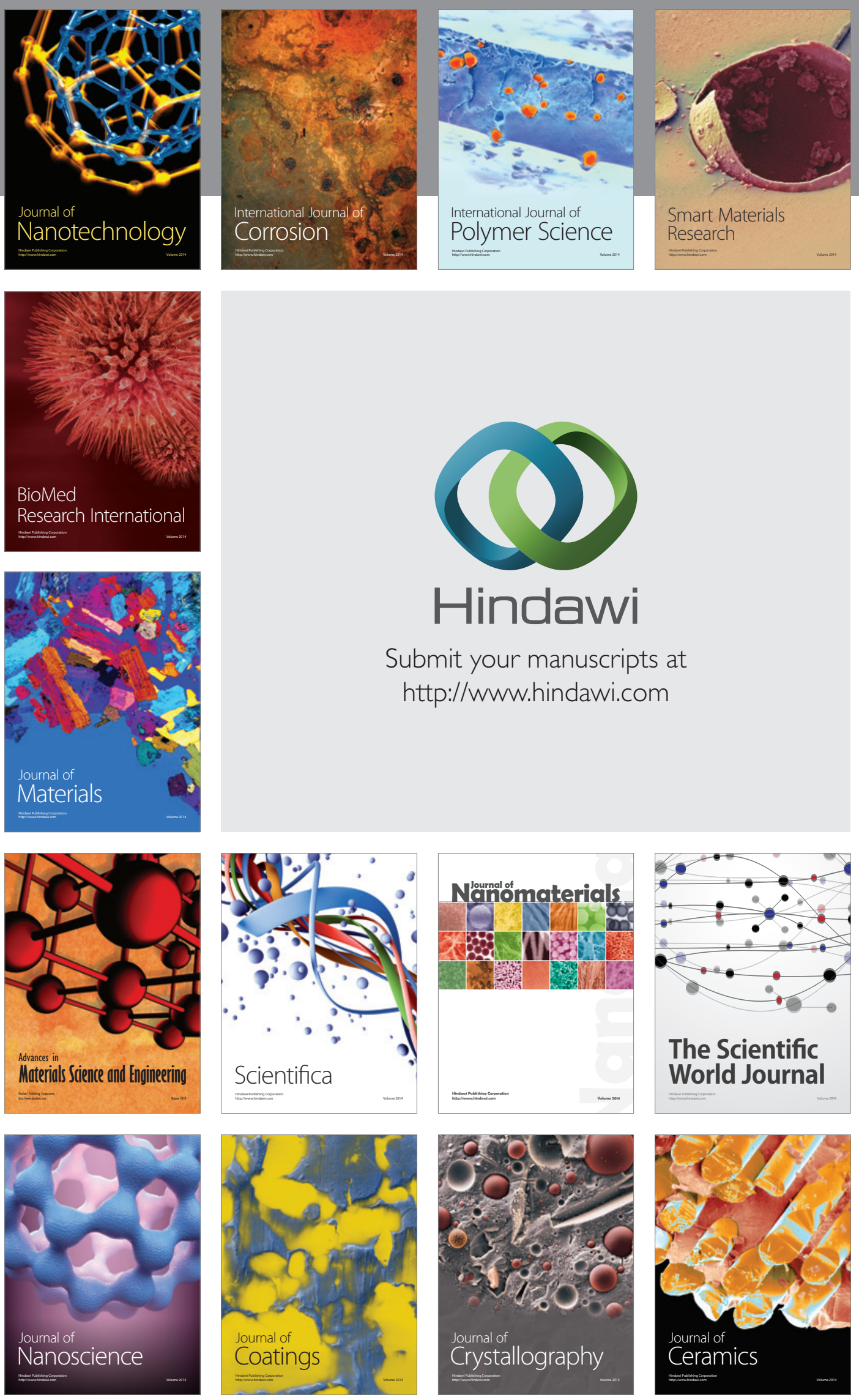

The Scientific World Journal

Submit your manuscripts at

http://www.hindawi.com

\section{World Journal}

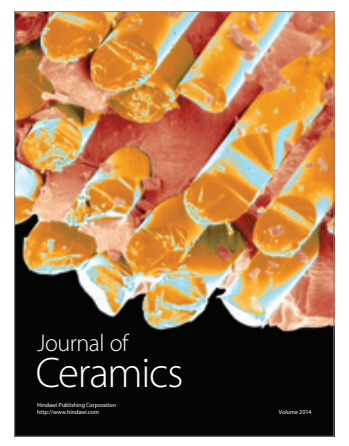

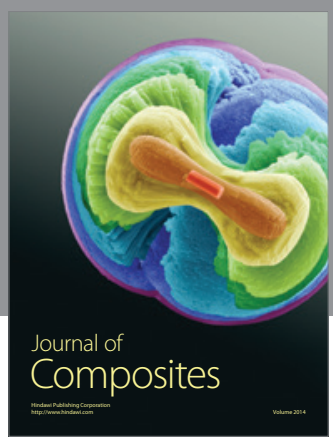
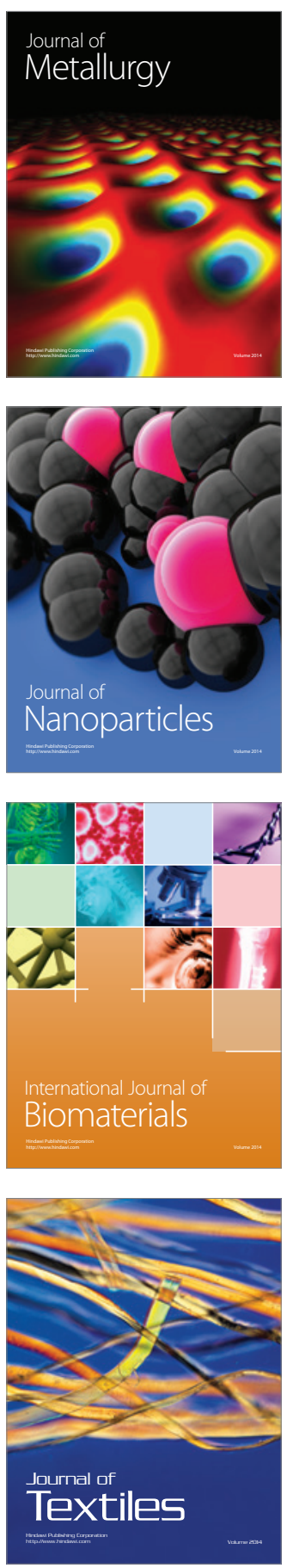